\title{
OUTILS DE DÉVELOPPEMENT DURABLE POUR L'INGÉNIEUR. COURS OFFERT AUX ÉTUDIANTS DU BACCALAURÉAT EN GÉNIE À L'ÉCOLE DE TECHNOLOGIE SUPÉRIEURE
}

\author{
Philippe Terrier, ing., M.ing,DESS gestion et développement durable \\ Maître d'enseignement à l'École de technologie supérieure \\ philippe.terrier@etsmtl.ca
}

\begin{abstract}
Résumé -Depuis l'été 2012, l'École de technologie supérieure (ÉTS) offre un cours en développement durable aux étudiants de $1^{\text {er }}$ cycle en ingénierie. Ce cours désigné par l'acronyme et le titre "ING 500: outils de développement durable pour l'ingénieur», vient compléter l'offre de cours optionnels dans presque tous les programmes de génie proposés à l'ÉTS. Le cours est conçu de façon à donner aux futurs ingénieurs des compétences multidisciplinaires en lien avec le développement durable (économie, droit de l'environnement, responsabilité sociale, analyse de cycle de vie, biomimetisme, gestion environnementale, éco conception...). Ce cours prépare également les étudiants à l'intégration du développement durable dans leur projet de fin d'études en leur présentant les outils indispensables $d u$ domaine. Cet article exposera la genèse du cours ING 500, son contenu ainsi que son organisation.
\end{abstract}

Mots clés: Développement durable, ingénierie, formation

\section{INTRODUCTION}

L'École de technologie supérieure à adopté en 2008 une politique de développement durable[1]. De cette politique a découlé un premier plan d'action 20092012[2] qui priorisait l'intégration du concept de développement durable dans les activités d'enseignement et de recherche. Cet engagement devait se concrétiser entre autres par la création d'un cours offert aux programmes de baccalauréat en ingénierie ( $1^{\mathrm{er}}$ cycle).

Afin de répondre aux engagements pris dans le cadre du plan d'action 2009-2012, un cours spécifiquement adapté aux exigences de la formation d'ingénieur offerte à l'ÉTS à donc été développé. Depuis l'été 2012, ce cours désigné par l'acronyme et le titre ING 500: outils de développement durable pour l'ingénieur est offert au sein des cours optionnels proposés à tous les programmes de génie (sauf génie de la construction).

Le travail d'élaboration complet du cours à nécessité environ 1000 heures. Effectué par un maître d'enseignement formé en ingénierie et développement durable le travail de développement du cours consistait à :

- sélectionner le contenu à présenter en tenant compte des concepts incontournables à ne pas manquer,

- répondre aux attentes des différents programmes envers les notions de développement durable,

- tenir compte du bagage de connaissances acquises par les étudiants avant de suivre le cours,

- positionner le cours au sein du cheminement de l'étudiant dans son programme de premier cycle à l'ÉTS,

- rédiger les notes de cours,

- préparer les présentations de cours,

- élaborer les exercices et activités pratiques qui seront réalisés chaque semaine,

- concevoir des projets de session concrets et pertinents.

Ce cours article exposera la genèse du cours ING 500, son contenu ainsi que son organisation.

\section{CONTENU DU COURS DE DÉVELOPPEMENT DURABLE POUR INGÉNIEUR}

Le cours ING 500 : outils de développement durable pour l'ingénieur est conçu de façon à donner aux futurs ingénieurs des compétences multidisciplinaires en lien avec le développement durable, un tout indivisible, mais qui peut être approché par l'étude séparée de ses 
différentes composantes, et qui doit être appliqué comme un ensemble de concepts inter reliés.

Le cours ING 500 a pour objectifs de présenter aux étudiants :

- les principes généraux de développement durable,

- le rôle de l'ingénieur dans les dossiers de développement durable,

- les outils de développement durable pour l'ingénieur,

- les compétences transversales nécessaires au dialogue avec toutes les parties prenantes multidisciplinaires.

Le tableau 1 présente un aperçu du contenu du cours exposé durant une session de 13 semaines.

Tableau 1: Sommaire du contenu du cours ING 500

\begin{tabular}{|c|c|}
\hline Cours & Contenu \\
\hline 1 & $\begin{array}{l}\text { Développement durable, introduction et mise } \\
\text { en contexte }\end{array}$ \\
\hline 2 & $\begin{array}{l}\text { L'ingénieur citoyen et acteur du } \\
\text { développement durable }\end{array}$ \\
\hline 3 & $\begin{array}{l}\text { L'entreprise et le positionnement en } \\
\text { développement durable }\end{array}$ \\
\hline 4 & Initiatives en développement durable \\
\hline 5 & $\begin{array}{l}\text { Outils pour une ingénierie plus durable en } \\
\text { entreprise. Introduction }\end{array}$ \\
\hline 6 & $\begin{array}{l}\text { Outils pour une ingénierie plus durable en } \\
\text { entreprise. Analyse de cycle de vie }\end{array}$ \\
\hline 7 & $\begin{array}{l}\text { Outils pour une ingénierie plus durable en } \\
\text { entreprise. Système de gestion } \\
\text { environnementale }\end{array}$ \\
\hline 8 & Droit de l'environnement \\
\hline 9 & Indicateurs de développement durable \\
\hline 10 & $\begin{array}{l}\text { Économie de la durabilité et de } \\
\text { l'environnement }\end{array}$ \\
\hline 11 & Responsabilité sociale de l'entreprise \\
\hline 12 & $\begin{array}{l}\text { La communication de la performance en } \\
\text { développement durable }\end{array}$ \\
\hline 13 & Gestion des risques et des catastrophes \\
\hline
\end{tabular}

L'élaboration du contenu du cours ING 500 est issue d'un processus de consultation tant interne à l'ÉTS qu'externe. En effet, le contenu du cours a été soumis à plusieurs représentants de divers départements au sein de l'ÉTS afin de valider la conformité envers leurs attentes. De plus, des rencontres avec des enseignants universitaires du domaine du génie et du développement durable mais externe à l'ÉTS ont été organisées afin de soumettre le contenu à la critique constructive des pairs.
Le cours vise également à préparer les étudiants à l'intégration du développement durable dans leur projet de fin d'études. De plus, fort de leur apprentissage et de leur découverte de nouveaux outils, les étudiants les plus intéressés peuvent déposer leur candidature pour le prix bâtir un monde durable avec génie[3] qui vise à récompenser le projet de fin d'études intégrant le mieux les aspects du développement durable. Ce prix de $1500 \$$ est remis annuellement par le décanat des études de l’ÉTS.

\section{ORGANISATION DU COURS DE DÉVELOPPEMENT DURABLE POUR INGÉNIEUR}

L'enseignement du cours ING 500 se compose d'une séance hebdomadaire d'une durée de trois heures, au cours de laquelle sont présentés des exposés magistraux et des activités encadrées. De plus, chaque semaine une séance de travaux pratiques et de laboratoires d'une durée de deux heures permet d'illustrer la matière vue en cours avec l'utilisation de logiciels, la résolution d'exercices, la tenue de débats, la venue de conférenciers, le visionnement d'extraits de documentaires. Durant certaine semaine, une période est allouée pour l'avancement du projet de session en équipe.

Les projets de session proposés aux étudiants et réalisés en équipes correspondent à des besoins réels, exprimés par des «partenaires» du cours. Ainsi, le bureau $d u$ développement durable de l'ÉTS[4] et l'ONG ingénieurs sans frontières Québec [5] alimentent régulièrement la banque de projets soumis aux étudiants chaque session. Afin d'offrir un large choix, qui réponde aux goûts de chaque équipe, au moins 5 projets de session sont proposés. Le jury d'évaluation des projets est composé, entre autres, de membres de l'organisme «partenaire» ayant proposé le projet.

\section{CONCLUSION}

Le cours ING 500 : outils de développement durable pour l'ingénieur a été créé à l'École de technologie supérieure afin d'honorer les engagements pris dans le cadre du plan d'action découlant de la politique de développement durable adoptée en 2008.

Le développement de ce cours a nécessité plus de 1000 heures de travail afin de composer un contenu répondant aux attentes des différents programmes offerts à l'ÉTS ainsi qu'aux exigences légitimes des étudiants. La 
popularité croissante du cours nous indique que nous avons bien répondu aux espérances des diverses parties prenantes.

À l'issue du cours ING 500: outils de développement durable pour l'ingénieur, les étudiants sont en mesure d'utiliser les principaux outils de développement durable pour l'ingénieur ainsi que de définir le rôle de l'ingénieur dans l'opérationnalisation du développement durable. Ils ont également développé une expertise leur permettant d'élaborer des recommandations et de développer un plan d'action pour implanter une démarche de durabilité dans l'entreprise et la société.

Offert depuis maintenant 1 an, le cours a connu un succès croissant et le nombre d'étudiants qui s'y sont inscrits a doublé entre l'été 2012 et l'hiver 2013.

\section{Références}

[1]. École de technologie supérieure: Politique de développement durable, 2008.

Consulté le 5 juin 2013. Disponible en ligne http://www.etsmtl.ca/A-propos/Direction/Politiquesreglements/Developpement_durable.

[2]. École de technologie supérieure: Plan d'action de développement durable.

Consulté le 5 juin 2013. Disponible en ligne http://www.etsmtl.ca/Services/dd/Engagement-de-lETS/Plan-d-action/ETS_PADD-2009-2012.pdf.

[3]. École de technologie supérieure: Prix Bâtir un monde durable avec génie.

Consulté le 5 juin 2013. Disponible en ligne http://www.etsmtl.ca/Etudiantsactuels/Baccalaureat/Vie-etudiante/Prixconcours/Prix_DD.aspx.

[4]. École de technologie supérieure: Bureau $\boldsymbol{d u}$ développement durable.

Consulté le 5 juin 2013. Disponible en ligne http://www.etsmtl.ca/Services/dd/Engagementde-l-ETS/Bureau-du-developpement-durable.

[5]. Ingénieurs Sans frontières Québec.

Consulté le 5 juin 2013. Disponible en ligne www.isfq.qc.ca. 